\title{
Covid-19 Virtual visiting and other technological adaptations for critical care
}

\author{
Authors: Hannah Webb, ${ }^{\mathrm{A}}$ Mark Parson, ${ }^{\mathrm{B}}$ Luke E Hodgson ${ }^{\mathrm{C}}$ and Khalil Daswani ${ }^{\mathrm{D}}$
}

\begin{abstract}
Social distancing measures introduced in response to the COVID-19 pandemic resulted in an almost complete cessation of family bedside interaction, with negative effects on patients, families and staff. Here we report on measures introduced in response to this situation at the critical care unit in one hospital involving the use of videoconferencing technology. The solutions used also had the potential to be extended to clinical use, for example when seeking advice for colleagues, and to the provision of training.
\end{abstract}

KEYWORDS: virtual visiting, telecommunications, critical care, COVID-19, communicating with relatives

DOI: $10.7861 /$ fhj.2020-0088

\section{Problem}

The pandemic caused by severe acute respiratory syndrome coronavirus 2 (SARS-CoV-2) led to a lockdown in the UK on the 23 March 2020 in an attempt to reduce transmission rates. Subsequent social distancing measures introduced by the UK Government also resulted in an almost complete cessation of friends and family visiting patients in NHS hospitals. Family bedside interaction is integral to the care we offer in the intensive care unit (ICU), and our team recognised that its loss would not only be heartbreaking for families, but also incur morale injury to staff. This is a short report of our iterative response to this situation, at the critical care unit of Worthing Hospital on the south coast of England.

\section{Potential solutions}

The structural adaptation of Worthing ICU was to temporarily expand from a single 12-bed unit into three units, caring for up

Authors: Aacute care common stem trainee, Worthing Hospital, Western Sussex Hospitals NHS Foundation Trust, Worthing, UK; ${ }^{B}$ anaesthetic registrar, Worthing Hospital, Western Sussex Hospitals NHS Foundation Trust, Worthing, UK; ' $\mathrm{ICM}$ and respiratory medicine consultant, Worthing Hospital, Western Sussex Hospitals NHS Foundation Trust, Worthing, UK, and visiting senior lecturer, University of Surrey, Guildford, UK; 'D core anaesthetic trainee, Worthing Hospital, Western Sussex Hospitals NHS Foundation Trust, Worthing, UK to 40 patients. Twice-daily ward rounds were typically covered by separate teams and both of these were followed by a doctor-led 'relative telephone round'. The named next of kin contact would receive a comprehensive update on the previous 24 hour's events, with opportunities to ask questions, and was then advised to disseminate information as they felt appropriate. This daily communication also took the burden off the nursing staff, who found it difficult to communicate while wearing personal protective equipment (PPE).

We conducted a purposive and multidisciplinary staff survey targeting 45 members of staff including physiotherapists, nurses (across experiences), healthcare assistants and doctors (including trainees and consultants) - to identify whether communication between staff and relatives was a problem, and if there were potential solutions to pursue.

From the 28 responses ( $62 \%$ response rate) we distinguished two key themes among the solutions proposed: video calling and/ or reserving a room for face-to-face meetings. Given video calling would allow compliance with the UK Government social distancing guidelines, we decided to pursue this option further. Guidelines from the Information Commissioner's Office confirmed that video calling would not be a breach of data protection laws ${ }^{1}$ and NHSX released guidance encouraging the use of mobile devices to prevent total isolation from loved ones. ${ }^{2}$ Following collaboration with our Information Management and Technology team, four iPads were purchased (one to each of the three critical care areas and one in the doctor's office), and restricted to Facetime software (Apple, Inc). Relatives were offered the opportunity to have a 'virtual visit' through a video call. If accepted, a preparatory explanation of what would be seen was given to the relative in an attempt to minimise any distress. Afterwards staff on the unit in PPE would call with the iPad at the bedside.

\section{Results so far}

From mid-March to June there have been approximately 350 video calls made from all four iPads. These do not include patients using their own devices. We conducted a second staff survey three weeks into implementing the tablets and also noted feedback from families and patients. Of the 45 members of staff emailed, 29 responded $(64 \%)$. Many families had requested daily video calls and feedback was very positive, one particular relative expressing 'it has been a lifeline', and relatives were largely sympathetic to the reasons behind the strict no-visiting policy. The first virtual visits had frequently been an intensely emotional process, especially when a patient had deteriorated quickly, 
needed intubation and had not been able to say a last goodbye. One member of staff reflected that 'it was perhaps at times more emotionally heavy than when on a normal day you' $d$ be with the relative at the bedside. However, it was amazing to be able to provide any kind of link for them to their sick loved one.' One patient has been able to feedback their rehabilitation experience having been ventilated for weeks: 'Using Facetime though has been amazing, especially seeing my two little granddaughters. It's such a great idea from the hospital to help keep our spirits high.'

Unforeseen benefits of video calling have included more of a collective interaction with families 'visiting' awake patients. This is because a group of people are able to 'dial in' and look at the device at the same time; rather than a single point of contact 'top-down dissemination model' with a phone call, there is more of a social group dynamic. Families have also visualised the staff they have been talking to over the phone, facilitating rapport and confidence that the patient is being cared for. That interaction has also been a morale boost for staff, particularly when they enable those first interactions following extubation.

As with all technological innovations, challenges have emerged. The usual visual cues of deterioration normally seen by visiting relatives - patients looking more tired or even the need for mechanical ventilation - are sometimes challenging for the doctor to convey, and for the next of kin to understand, over the telephone. These conversations cannot universally prepare someone for the actual change in appearance of their loved one. An example of this was when one relative became acutely distressed seeing their next of kin for the first time since admission, and subsequently our staff reported it can feel difficult via video calling to comfort someone as they naturally would do face-to-face.

The moral injury staff may incur from overhearing often intimate and emotional conversations is not to be underestimated. Although privacy of conversations at the bedside is difficult under normal circumstances, there are other elements that are harder to control. The volume of the tablets sometimes had to be high to be heard over the sound of CPAP, and relatives were unable to see 360 degrees to know when staff are in the cubicle. This also impacted on confidentiality in open bays, as patients in adjacent cubicles may hear through curtains or that the person virtually visiting may hear or see something that breaches someone else's privacy. Staff were alerted to this and were careful to manage the volume and direction of the iPad, so that privacy was respected.

Recording consultations has been helpful for patients and visitors prior to the pandemic; however, all participants must consent and recordings can be made without the clinicians' awareness. Some of these challenges have been addressed in the Intensive Care Society guidance. However, they state that if a patient cannot participate themselves, then each call made has to be assessed to be in their best interests. ${ }^{3}$ This doesn't draw the parallel that visitors would normally come to sit at the bedside of sedated patients, without clear evidence that the patient would want visitors. We do not routinely ask if patients would want their family to be in the room during their cardiac arrest resuscitation; it is acknowledged that families are also owed our care and that it is their choice too.

It was important to make setting up a virtual visit easy for the whole multidisciplinary team, many of whom had not previously worked with us on ICU before. We developed a quick reference guide to illustrate the process whilst allowing relatives, patients and staff to troubleshoot most basic technical difficulties faced while trying to set up a video call. This was of particular help to the less technologically literate who may have seen this as a barrier to engagement.

Use of the proprietary Facetime software unfortunately limited video calls to relatives with Apple products only. We initially overcame this issue by offering a visit to the Unit's 'Relatives Room' to communicate using the unit's 'clean' iPad. However, to improve the safety of those visiting and to include as many users as possible, discussions into the use of non-proprietary video communication software are ongoing. The decision to utilise Facetime was due to concerns about security with other systems, some of which have unfortunately been borne out. ${ }^{4}$

\section{Further evaluation}

The presence of tablets in all three units resulted in another adaptation during ward rounds. To minimise the number of staff needing to don PPE and make the ward round more efficient, one or two doctors would video call from the doctor's office and participate in a live stream of the ward round. They could react in real time by requesting investigations or making referrals while also scribing the round. Any visiting teams could communicate efficiently via these doctors in the hub of the doctors' office, without having to wait until the consultant had finished and doffed PPE. Examples of similar adaptations to ward rounds also include allowing medical students to join remotely and continue their learning. ${ }^{5}$

Our nursing colleagues could call the doctor's office iPad to request a quick visual review of ventilator settings, with the doctors able to give speedy advice, instead spending time donning of PPE. The staff survey, however, did reveal that occasionally nursing colleagues felt there was a decrease in the presence of doctors physically attending the unit.

An exciting development was adding the 'Cardmedic' application to the tablets. ${ }^{6}$ This was to facilitate communication with patients that would otherwise have challenging due to the barrier created by PPE and noisy CPAP hoods. Patients could read clear and large text flashcards on the tablet devices to better understand staff and to enable the consenting process for procedures.

Many clinicians and the public have become familiar with different video conferencing platforms during lockdown, and some have developed software to facilitate virtual visiting such as NHS AttendAnywhere ${ }^{7}$ and Lifelines ${ }^{8}$ that address some of the confidentiality issues.

\section{Potential future}

Video conferencing is by no means completely novel, as represented by published NHS Telehealth Monitoring standards available since $2010 .^{9}$ However the current pandemic has led to healthcare across the world adapting rapidly to find solutions while continuing to provide high quality care. Some of these solutions were vital, stopgap approaches that will pave the way for more streamlined and data secure technology..$^{10}$ General practice has led the field with remote assessment of patients and has highlighted the importance of visual clues gained within the assessment.11 This is akin to what we have seen in our daily practice, but on this occasion it is the families of the patients who are benefitting more from these visual clues. Emergency medicine services have trialled using iPads to virtually assess patients and improve overcrowding.12 In the context of ICU, we now have the potential to use video calling as part of rehabilitation and follow up clinics. The future 
expansion of telecommunications for virtual visiting and virtual clinics may help us towards a greener NHS. The implementation of these technologies results in fewer car journeys which in turn may reduce air pollution and the associated disease burden. ${ }^{13}$

We have all looked forward to a time where our patients and families can be reunited. That said, the rapid uptake of technology during this time is a huge step forward for telemedicine. Virtual visiting will have an ongoing role for those families who are at a distance or cannot otherwise attend hospital regularly. We hope other units will find our experience of use, with its relatively low-cost initial outlay and significant benefits to family and staff communication in the midst of the COVID-19 pandemic.

\section{References}

1 Information Commissioner's Office. ICO statement on data sharing during the Covid-19 pandemic. https://ico.org.uk/global/ data-protection-and-coronavirus-information-hub/data-protectionand-coronavirus/health-social-care-organisations-and-coronaviruswhat-you-need-to-know/ [Accessed 23 June 2020].

2 NHSX. Use of mobile devices by patients in hospitals. www.nhsX. nhs.uk/covid-19-response/data-and-information-governance/usemobile-devices-patients-hospitals-eg-phones-tablets-and-cameras/ [Accessed 23 June 2020].

3 UK Intensive Care Society. Guidance on the use of video communication for patients and relatives on ICU. www. acprc.org.uk/Data/Resource_Downloads/Covid19_

Guidanceontheuseofvideocommunucationforpatientsandrelative... pdf [Accessed 23 June 2020].

4 Paul K. 'Zoom is malware': why experts worry about the video conferencing platform. The Guardian, 2 April 2020.
5 Schwamm LH, Estrada J, Erskine A, Licurse A. Virtual care: new models of caring for our patients and workforce. Lancet Digital Health 2020:2:e282-5.

6 Grimaldi R. Cardmedic. Available from www.cardmedic.com/ [Accessed 3 May 2020].

7 NHS England. Attend Anywhere. Available from https://england. nhs.attendanywhere.com/resourcecentre/Content/Home.htm [Accessed 24 June 2020].

8 Kings's Health Partners. Life Lines. Available from www.kingshealthpartners.org/our-work/lifelines [Accessed 24 June 2020].

9 NHS Clinical Protocol. Telehealth monitoring standards 2010. Avaliable from https://www.england.nhs.uk/wp-content/ uploads/2014/12/Telehealth-Monitoring-Standards.pdf [Accessed 28 April 2020].

10 Wosik J, Fudim M, Cameron B et al. Telehealth transformation: COVID-19 and the rise of virtual care. J Am Med Inform Assoc 2020;27:957-62.

11 Greenhalgh T, Koh G, Car J. Covid-19: a remote assessment in primary care. BMJ 2020;368:m1182.

12 Wittbold KA, Baugh J], Yun BJ et al. iPad deployment for virtual evaluation in the emergency department during the COVID-19 pandemic. Am J Emerg Med 2020, in press (doi: 10.1016/j. ajem.2020.04.025).

13 Holmner A, Ebi KL, Lazuardi L, Nilsson M. Carbon footprint of telemedicine solutions - unexplored opportunity for reducing carbon emissions in the health sector. PLOS ONE 2014;9:e105040.

Address for correspondence: Dr Hannah Webb, Worthing and Southlands Hospitals NHS Trust, Lyndhurst Road, Worthing BN11 2DH, UK.

Email: hannahjmwebb@outlook.com 